\title{
Engineering reversible elasticity in ductile and brittle thin films supported by a plastic foil
}

\author{
Nicolas Vachicouras ${ }^{1}$, Christina M. Tringides ${ }^{1}$, Philippe B. Campiche ${ }^{1}$, \\ Stéphanie P. Lacour* \\ Bertarelli Foundation Chair in Neuroprosthetic Technology, Laboratory for Soft Bioelectronic Interfaces, Institute of Microengineering, Institute of \\ Bioengineering, Center for Neuroprosthetics, École Polytechnique Fédérale de Lausanne (EPFL), 1202 Geneva, Switzerland
}

\section{A R T I C L E I N F O}

\section{Article history:}

Received 21 March 2017

Received in revised form 30 May 2017

Accepted 30 May 2017

Available online 6 June 2017

\section{Keywords:}

Kirigami

Thin films

Electronic materials

Engineered elasticity

\begin{abstract}
A B S T R A C T
Reversible deformation is a unique property of elastic materials. Here, we design and fabricate highly stretchable multilayered films by patterning Y-shaped motifs through films of non-elastic materials, e.g. plastics, metals, ceramics. By adjusting the geometry and density of the motif, as well as the thickness of the film(s), the effective spring constant of the engineered film(s) can be tuned. Three-dimensional bending of the patterned film(s) enables macroscopic stretchability and minimizes local film strain fields. The engineered films demonstrate no preferential direction of stretching and the proposed design is versatile. Furthermore our approach is compatible with thin-film processing. We demonstrate the Yshaped motifs allow for the design of stretchable plastic foils coated with metallic and metal oxide conductors. We anticipate the patterned motifs can be scaled down to offer a wider range of elastic electronic materials to use in stretchable electronics and to create soft bioelectronics.
\end{abstract}

(c) 2017 Elsevier Ltd. All rights reserved.

\section{Introduction}

Stretchable electronics adapt traditionally rigid circuits to three-dimensional and dynamic shapes [1]. The human body can be fitted with epidermal physiology sensors, wearable displays or conformable neural implants [2].

Elastic electronic materials display stable and reliable electrical properties upon repeated and multi-axial deformations [3,4]. An alternative approach to produce stretchable devices and circuits out of non-elastic materials is the patterning of strain-relief motifs in the form of serpentines, to minimize local mechanical stress in the most fragile materials $[5,6]$. The advantage of such an approach is compatibility with standard microfabrication techniques and materials. Most reported designs include freestanding meandering patterns that host complete devices or circuits, e.g. metallic interconnects, silicon strain sensors, MOS transistors or light emitting diodes. Thus, the approach of serpentine patterns adds a significant circuit layout constraint, especially when preparing matrices of interconnected devices.

A Kirigami approach, based on the Japanese art of cutting paper, has been recently used to pattern thin films with arrays of cuts which enable large deformations through direct buckling and folding processes [7-9]. Parallel slits patterned in parylene films and

\footnotetext{
* Corresponding author.

E-mail address: stephanie.lacour@epfl.ch (S.P. Lacour).

1 These authors contributed equally to this work.
}

graphene oxide/polyvinyl alcohol (GO-PVA) nanocomposite films allow for high, reversible deformability [10]. Interestingly, a similar strain relief concept is observed in thin gold films deposited on silicone membrane, which form spontaneous micro-cracked patterns $[11,12]$. The later usually have three "branches" (forming Yshaped motifs) and are randomly distributed over the film surface area. Upon stretching, minute deformation occurs in the thin gold ligaments as they deflect out-of-the-plane, thereby preserving the electrical conductivity of the metal film [13].

Inspired by these Y-shaped motifs observed on micro-cracked gold films, we propose to engineer such a "three-branched motif" in non- or minimally-elastic materials and expand the list of stretchable thin film materials. Moreover, these Y-shaped cuts are a natural step following the recently proposed Kirigami horizontal slit patterns [10]. In addition, we reasoned the Y-shape enables elasticity without dramatically decreasing the electrical resistance of the conductor. We focus on a macroscopic design to facilitate observations on stretchability, but we anticipate the scalability of our design to micro- and even nano-scales. The cut motif has a Y-shape and is distributed in a hexagonal pattern throughout the entire surface of the film(s). The symmetry of the motif enables elasticity along any direction, a significant advantage to conform to objects with non-Gaussian curvature. We demonstrate our approach is compatible with plastic foil(s) but also allows for the design of stretchable multilayers composed of ductile and/or brittle thin film(s) supported by plastic foil(s). 

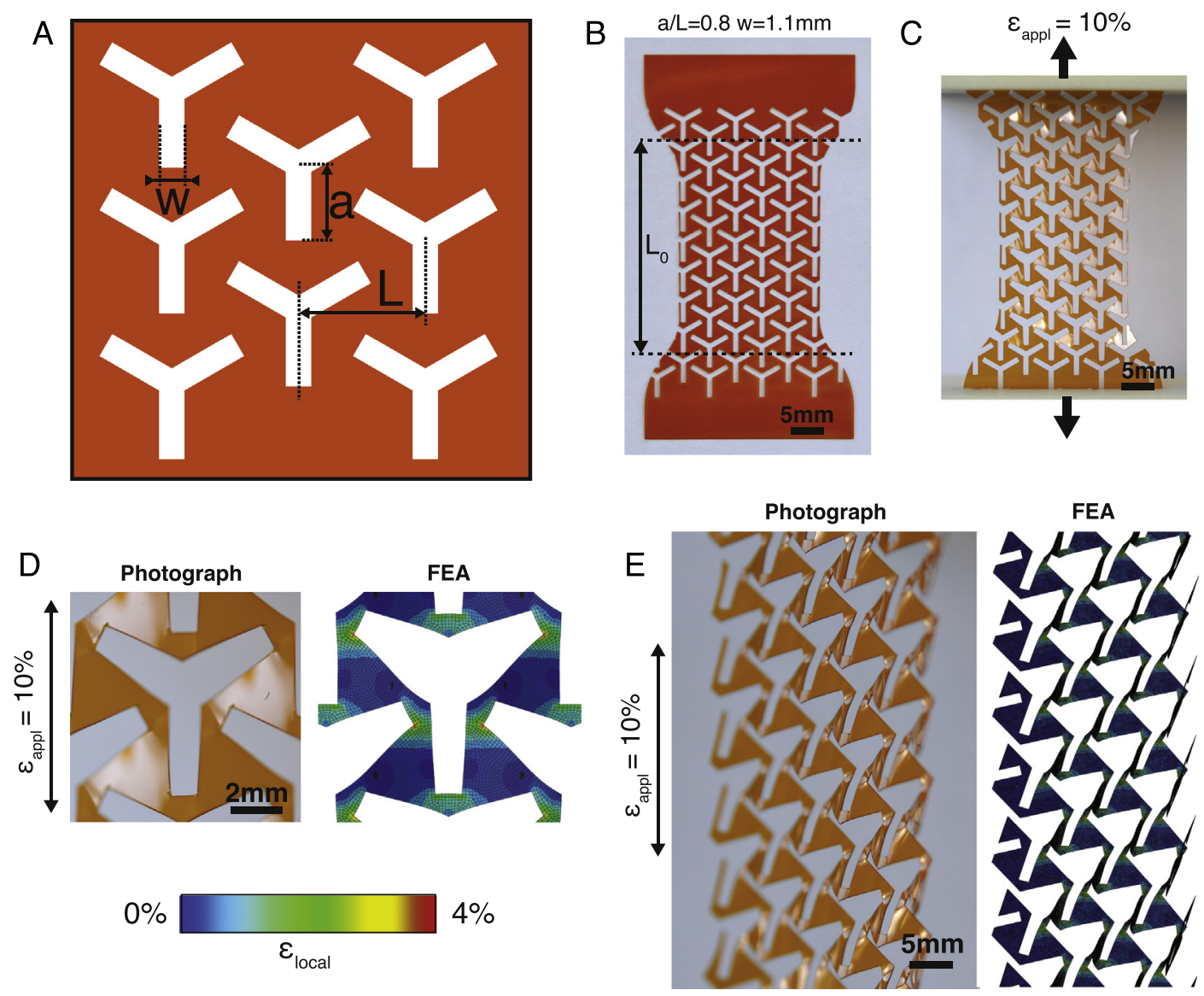

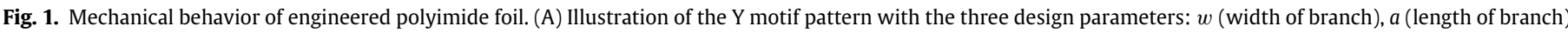

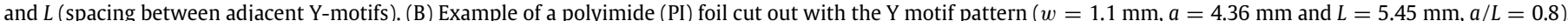

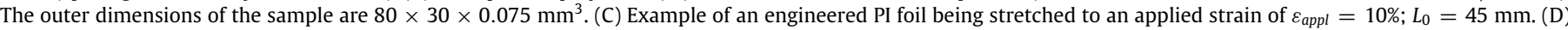

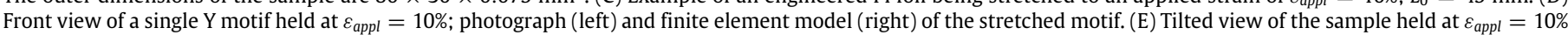

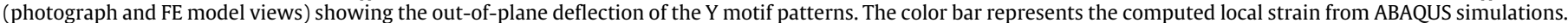
(For interpretation of the references to color in this figure legend, the reader is referred to the web version of this article.)

\section{Methods}

Sample preparation. Plastic carrier substrates, i.e. polyimide, Kapton ${ }^{\circledR}$ HN - Standard Rolls - 610 mm wide and PET (polyethylene terephthalate, Mylar ${ }^{\circledR}$ ) foils, were purchased from Lohmann Technologies. Both plastic foils were $75 \mu \mathrm{m}$ thick. Y-shaped patterns were cut into the foils with a Plotter Cutter (RoboPro CE500040-CRP) using a DXF file generated from a MATLAB (R2015b) script. The contour of a dogbone shape (norm DIN 53504, Suppl. Fig. S1) was overlayed on the cut film. The wider ends of the dogbone were left without cracks to allow for firm clamping of the sample in the tensile equipment, while the thinner central part was covered with the Y-shaped cuts that could stretch.

Thin titanium / platinum (Ti / Pt, 25 / $100 \mathrm{~nm}$ thick) films were evaporated by e-beam (Alliance-Concept EVA 760) on polyimide foils that were first activated using an oxygen plasma, in order to improve the metal films adhesion to the plastic. Indium tin oxide (ITO) films (100 nm in thickness) were sputtered (Pfeiffer SPIDER $600)$ on the PET foil.

PDMS membranes (poly(dimethylsiloxane), Sylgard 184, Dow Corning, mixed at 10:1 (w:w) with a pre-polymer crosslinker) were prepared by spin-coating on silicon $(\mathrm{Si})$ wafer. First, the Si wafer was coated with a self-assembled layer of trichloro $(1 \mathrm{H}, 1 \mathrm{H}, 2 \mathrm{H}, 2 \mathrm{H}-$ perfluorooctyl) silane (Sigma Aldrich) in a desiccator. Next, PDMS was spin-coated on the Si wafer (750 RPM for $35 \mathrm{~s}$ ) and cured at $80{ }^{\circ} \mathrm{C}$ for at least $3 \mathrm{~h}$ in a convection oven. The PDMS membrane was then cut out using the same technique as for the plastic foils and released manually from the wafer
Electromechanical characterization: All samples (except for bulk PI and PET) were stretched in a tensile tester (MTS Criterion Model 42 , with a load cell of $100 \mathrm{~N}$ maximum capacity) using a strain rate of $1 \mathrm{~mm} / \mathrm{s}$. The samples were clamped and secured to the plates of the stretcher with double-sided tape. The electrical resistance of the conductive films was recorded during stretching using a Keithley 2400 sourcemeter.

The bulk PI and PET were characterized in a $100 \mathrm{kN}$ MTS 809 axial-torsional servohydraulic testing machine with a load cell of $10 \mathrm{kN}$. The force-displacement data was measured at a sampling frequency of $100 \mathrm{~Hz}$ using the MTS TestSuite TW software and analyzed using MATLAB (R2015b).

For longer-term cycling, the samples were mounted to a homemade uniaxial stretcher and stretched at $10 \%$ of their initial length at $1 \mathrm{~Hz}$ ( 1 stretching cycle per second). The electrical resistance was measured using a 4-probe system and a Keithley 2400 sourcemeter. The stretcher and sourcemeter were both controlled using custom made software based on LabView 2015. The resistance was recorded (sampling rate of $5 \mathrm{~Hz}$ ) and saved only every 10 cycles per decade of cycles.

Scanning Electron Microscopy (SEM): SEM images were acquired with a Zeiss Merlin microscope using an annular detector at a beam energy of $1 \mathrm{keV}$.

Finite element analysis (FEA): Mechanical simulations were performed using ABAQUS (6.14) in order to evaluate the effect of the different geometries' parameter. The standard static procedure was used with a damping factor of $1 \mathrm{e}-7$. The S4R element was used with a global mesh size of $0.15 \mathrm{~mm}$ and no dependence on 

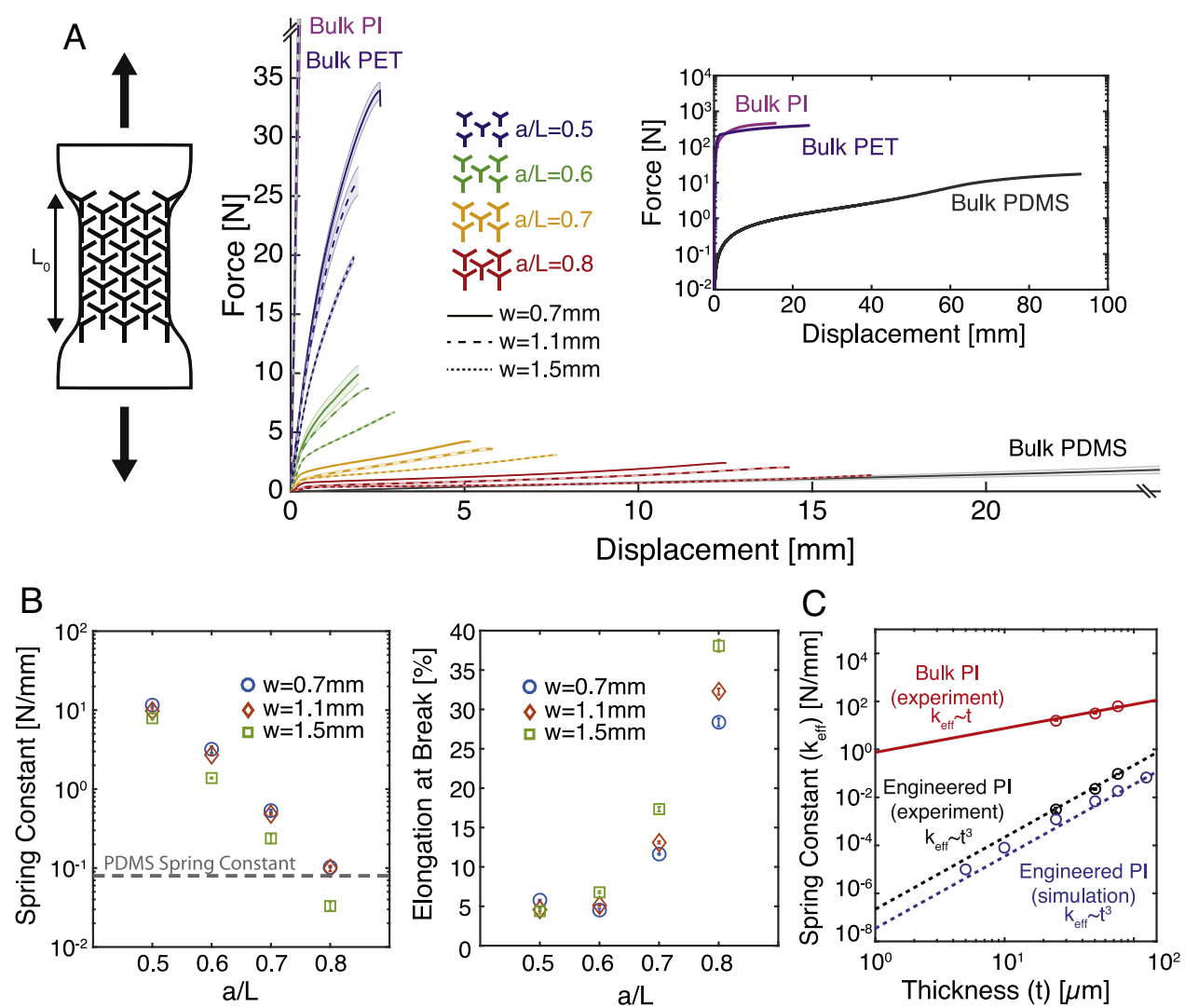

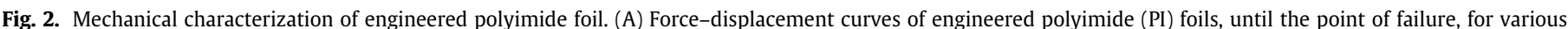

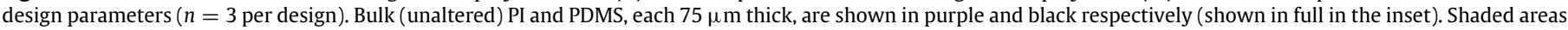

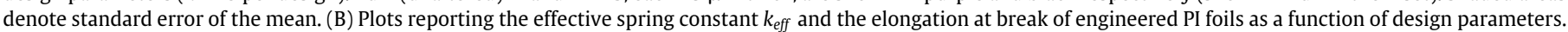

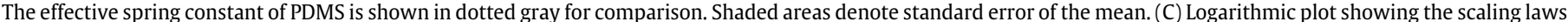

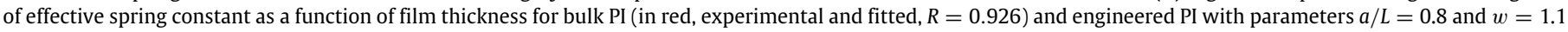

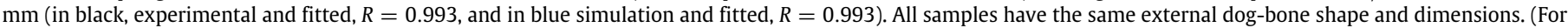
interpretation of the references to color in this figure legend, the reader is referred to the web version of this article.)

the mesh size was observed. Two steps were created with a first step of perturbations applied on three random points and in the direction perpendicular to the film's surface and clamped extremities. The influence of perturbations' magnitude was analyzed and no influence on the force-displacement behavior was observed. The second step consisted of a clamped boundary condition on one end and a displacement in the stretching direction on the other end while deactivating the perturbations.

Electrical simulations were performed with COMSOL (2015) in order to estimate the resistance of a $100 \mathrm{~nm}$ thick conductive sheet of Pt, which was cutout with various geometries of the Y-shaped pattern. The ground boundary condition was applied on one end of the sheet and a terminal with $1 \mathrm{~A}$ was applied to the other hand. The resulting maximum voltage field was used to estimate the resistance by dividing its value by $1 \mathrm{~A}$.

\section{Results}

Each of the three branches of a given $\mathrm{Y}$ motif had the same length (parameter " $a$ ") and width (" $w$ "), and each dogbone sample was patterned with the same Y motif. The Y motifs were distributed in a hexagon pattern with the distance between two motif centers, on the $x$-axis, named " $L$ " (Fig. 1(A)). Fig. 1(B) shows a photograph of a dogbone sample cut from the bulk PI foil. The length of the sample that underwent stretching and deflection was labeled $L_{0}$ (Fig. 1(B)). At 10\% applied strain, the PI ligaments bend, and deflect out-of-plane to locally relieve strain (Fig. 1(C)). This mechanical behavior of the sample under tensile strain was correctly predicted by FEA, which supplements the experimental results by offering an estimation of the local strain of various points along the branches. Experimental and FEA images of the stretched samples match remarkably well (Fig. 1(D)-(E), Suppl. Video V1).

\subsection{Effect of the geometry of the Y motif}

Twelve different combinations of $(a, w$, and $L)$ were cut and stretched until failure. We defined the failure strain as the strain at which the first motif broke or tore. We tested 4 ratios of $a / L$ $(0.5,0.6,0.7,0.8$, where we varied $a$ from $2.3 \mathrm{~mm}$ to $4.36 \mathrm{~mm}$, and $\mathrm{L}$ was kept constant at $5.45 \mathrm{~mm})$ and 3 values of $w(0.7 \mathrm{~mm}$, $1.1 \mathrm{~mm}, 1.5 \mathrm{~mm})$. Three samples of each combination were measured $(n=3)$. For comparison, $75 \mu \mathrm{m}$ thick samples cut in a dogbone shape (without Y motifs) of bulk PI, bulk PET and bulk PDMS were also stretched to failure. Fig. 2(A) displays all 15 forcedisplacement curves, with bulk carrier responses in the inset on a semi-logarithmic plot. As the width $w$ and $a / L$ ratio increase, the footprint of the plastic struts "shrinks" and the effective spring constant, $k_{\text {eff }}$, calculated as the slope of the linear region of the force-displacement curve (Fig. 2(B), left), decreases. A large $a / L$ ratio (of 0.8 ) patterned in the plastic foil enables matching of the PDMS spring constant. The width of the cuts, $w$, influences both $k_{\text {eff }}$ and the elongation at break (Fig. 2(B), center).

Based on these observations and ease of manipulation of the samples, we selected an $a / L$ of 0.8 and $w=1.1 \mathrm{~mm}$ for all subsequent experiments.

Next, we tested the effect of the foil thickness on the effective spring constant of the engineered structures (Fig. 2(C)). $k_{\text {eff }}$ scales with $t^{3}$, where $t$ is the thickness of the PI foil, indicating the 

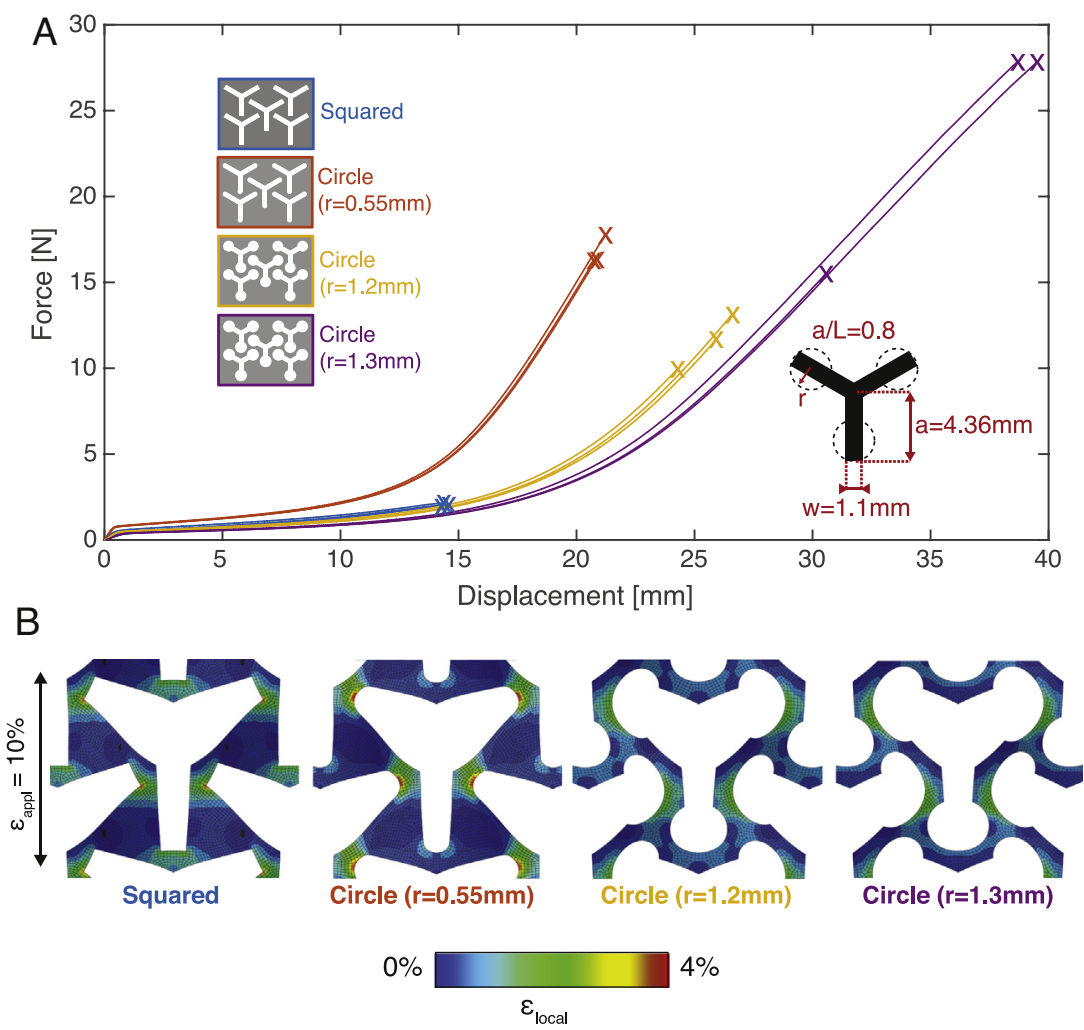

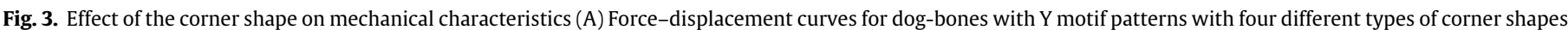

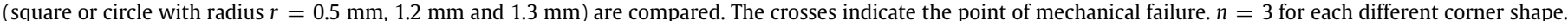

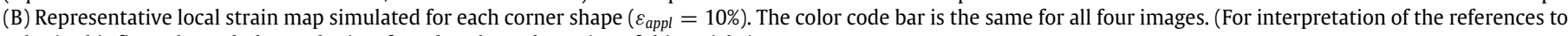
color in this figure legend, the reader is referred to the web version of this article.)

deflection and buckling of the PI struts dominate the macroscopic response of the engineered foil to elongation. Experimental and simulation data fit well. In comparison, $k_{\text {eff }}$ in the bulk PI linearly scales with $t$.

\subsection{Effect of the $Y$ branch corner shape}

Experiments were first completed with $\mathrm{Y}$ motifs that had squared $\left(90^{\circ}\right.$ angle $)$ corners at the end of each branch. We observed at this location the highest strain within the motif upon stretching. We then hypothesized that rounding the corners of each branch would increase the macroscopic strain to failure of the engineered foil by suppressing the location of crack initiation. Furthermore, the design of circular endings with a diameter larger than $w$ should further enhance the compliance of the foil. We compared four geometries: squared, rounded, circles with radius $1.2 \mathrm{~mm}$, and circles with radius $1.3 \mathrm{~mm}$ (Fig. 3). Our previous results showed that for a constant $Y$ length $a$, the shorter the distance between two cuts, the lower the effective spring constant. We thus kept that value constant (the total length of the branch, $a$, was always equal to $4.36 \mathrm{~mm}$ and the $a / L$ ratio was kept at 0.8 ). The rounded design simply substitutes the sharp $90^{\circ}$ angle to a quarter-circle. Complete circles ( $1.2 \mathrm{~mm}$ or $1.3 \mathrm{~mm}$ radius) were added such that the total length of each branch $a$ was unchanged, as indicated in a schematic Fig. 3(A). Because the branch length and $a / L$ ratio were the same for all samples, the spring constant of the four types of samples was relatively unchanged. However, we observed a significant increase in the strain at failure for samples with circular endings. FEA simulation confirmed the maximum local strain decreases as circles of larger diameter terminate the Y motif branches (Fig. 3(B)). Furthermore, upon tensile loading, the strain is more uniformly distributed throughout the struts of PI in-between the Y motifs with circular endings.
Subsequent characterization was therefore conducted on engineered foils carrying Y motifs with $1.3 \mathrm{~mm}$ radius circular endings, and a geometry of $a / L=0.8$ and $w=1.1 \mathrm{~mm}$.

\section{Applications to the design of stretchable thin film conduc- tors supported by a plastic substrate}

\subsection{Stretchable ductile metal film on polyimide foil}

Engineered polyimide dogbones prepared as described in the previous section were coated with a Ti/Pt bilayer. We evaluated the elasticity of the construct as a function of the electrical resistance of the metallic conductor. The highest electrical resistance is observed for the most compliant motif design (Fig. 4(A)). For the widest $w$, with an $a / L$ of 0.8 , the resistance is almost 3 times higher than for an $a / L$ of 0.5 with the same $w$. We used these results to decide on an optimized electro-mechanical tradeoff i.e. high elasticity, low electrical resistance, with Y motifs of $w=1.1 \mathrm{~mm}$, $a / L=0.8$.

Fig. 4(B) illustrates the electro-mechanical behavior of a $\mathrm{PI} / \mathrm{Pt}$ construct with Y motifs with circular endings. The samples sustained uni-axial stretching until $70 \%$ strain before mechanically failing. The resistance remained remarkably constant until $40 \%$ strain indicating the engineered motifs provide efficient strain relief to the metallic films.

Using a radial stretcher [14], we mounted a $10 \mathrm{~cm}$ diameter disk of engineered PI onto which a rectangular strip of Pt was patterned along the disk diameter, and we then stretched the construct. The width of the conductor matched that of a Y motif $(\approx$ $2 \mathrm{a})$. With an increase of $40 \%$ in surface area, the Pt on engineered PI remained conductive (Suppl. Fig. S2). The symmetry of the Y motif enables multi-axial deformation (Suppl. Video V2). We next evaluated the robustness of the $\mathrm{PI} / \mathrm{Pt}$ construct as a function of 

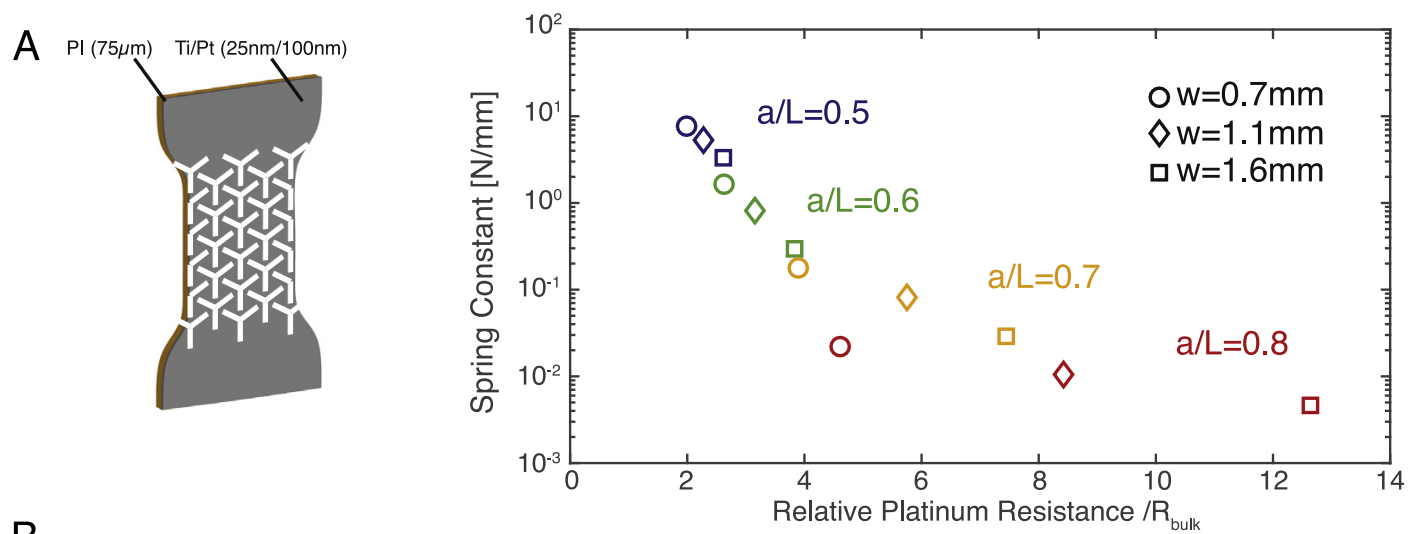

B

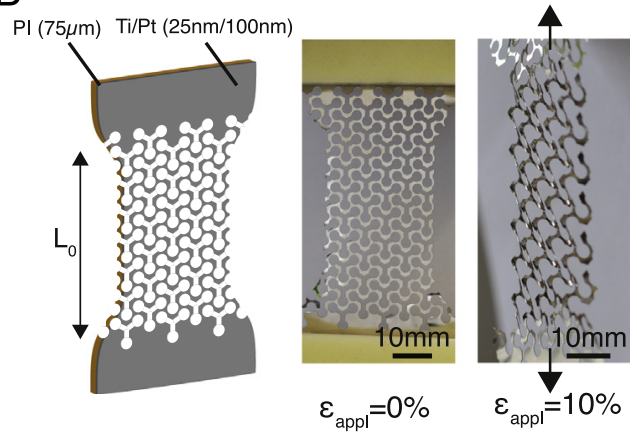

D

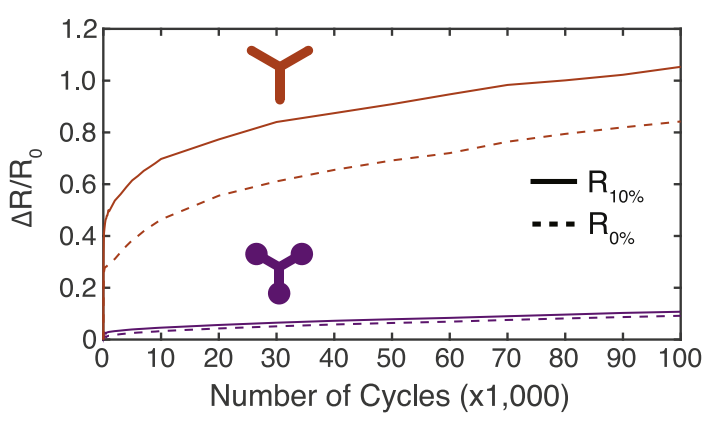

$\mathrm{C}$
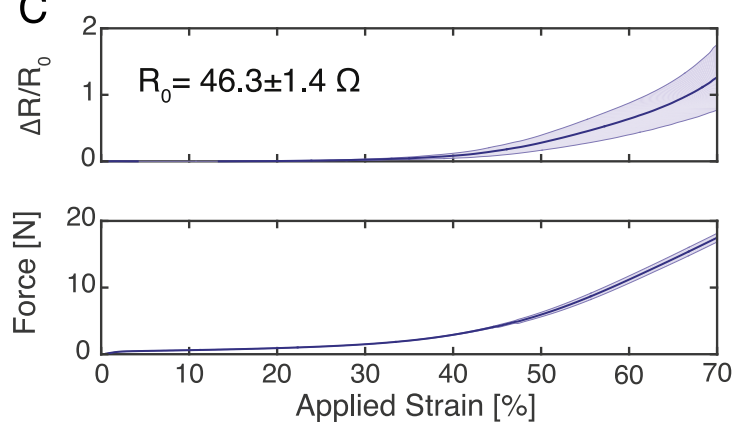

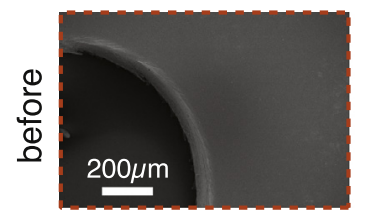

0 cycles

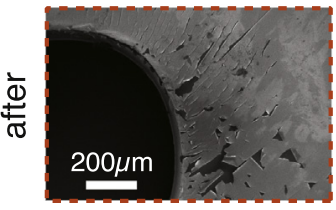

100k cycles

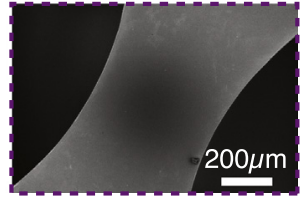

0 cycles

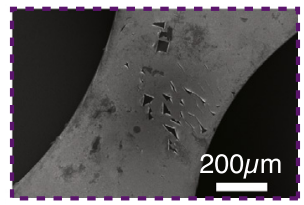

$100 \mathrm{k}$ cycles

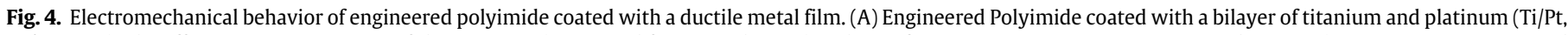

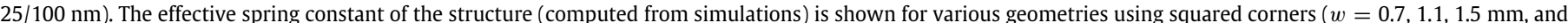

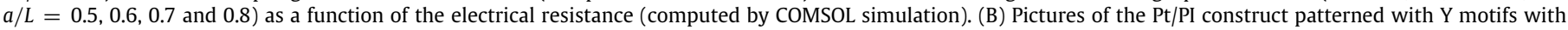

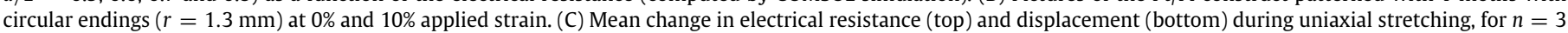

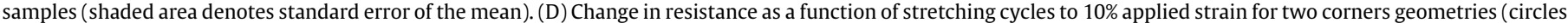

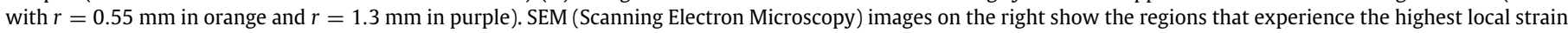

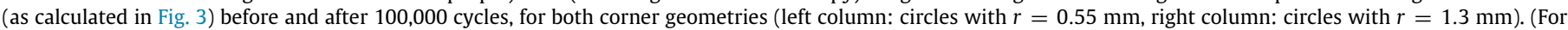
interpretation of the references to color in this figure legend, the reader is referred to the web version of this article.)

significant mechanical cycling at $10 \%$ strain. We stretched samples with rounded end $\mathrm{Y}$ motifs and motifs with circular endings each for 100,000 cycles (Fig. 4(C)) and compared their behaviors. Both designs produced highly stretchable conductors. However samples prepared with $\mathrm{Y}$ motifs with circular endings displayed higher performance in terms of less resistance change with strain and improved resistance stability over time, with a resistance increase of $\sim 10 \%$ after the 100,000 cycles.

After cycling, we imaged using SEM the motif areas where FEA simulation predicted the highest strain. Cracks and delamination were clearly observed in these regions (Fig. 4(C), right) while the rest of the sample was crack-free (Suppl. Fig. S3). Following 1000 stretching cycles, little damage is observed in the platinum film (data not shown). After 100,000 cycles, we observed short cracks formed during the repeated loading/unloading cycles, and local delamination of the metal film from the polyimide substrate in the regions of the highest strain (as predicted by the FEA). The overall conductor maintained however continuous electrical conductivity. The rounded end samples displayed significantly more delamination of the Pt film from the PI carrier as compared to the samples prepared with motifs with circular endings, further highlighting the importance of the motif design.

\subsection{Stretchable, transparent, brittle metal oxide films on engineered} PET

Next, we tested our Y motif design to produce stretchable transparent conductors from brittle indium tin oxide (ITO) films. While metals such as Pt can sustain some plastic deformation before fracture, the critical failure strain of metal oxides is not higher than $1.5 \%$. 

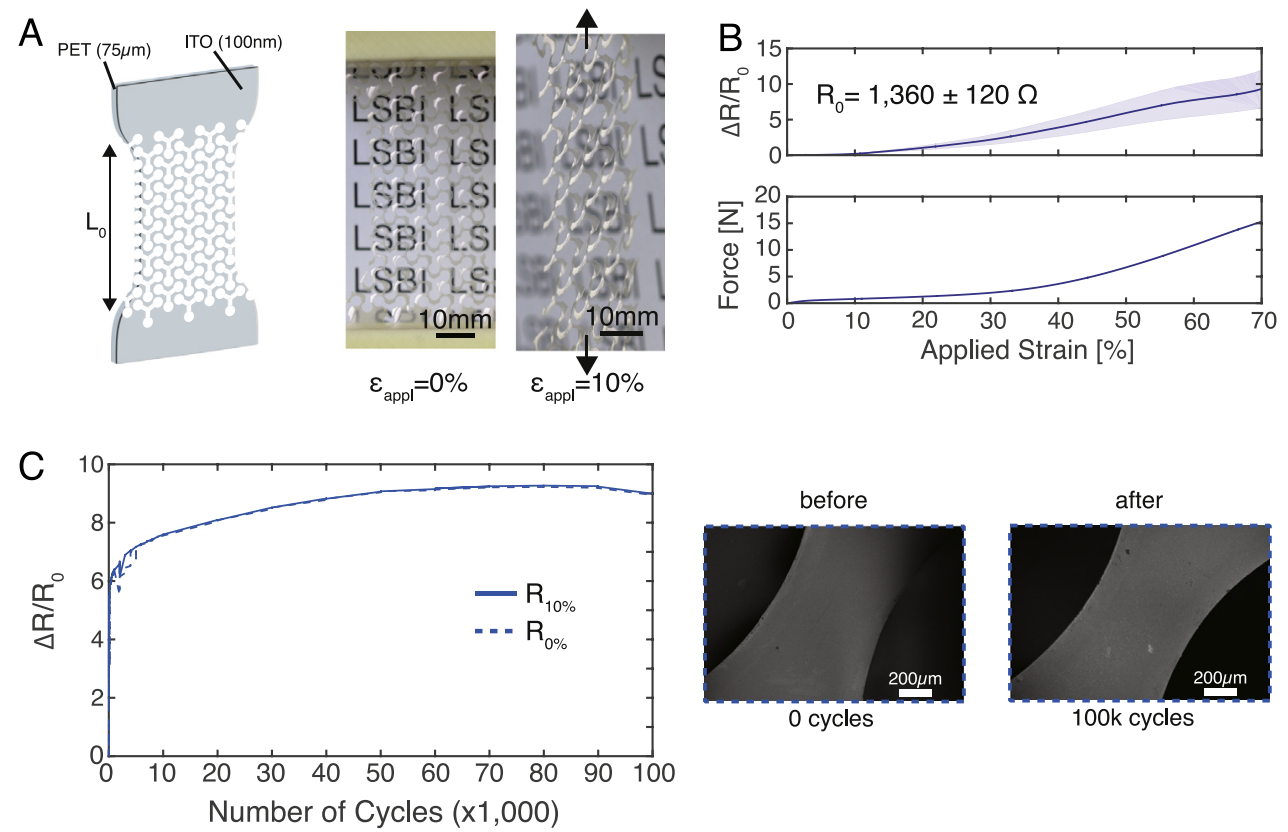

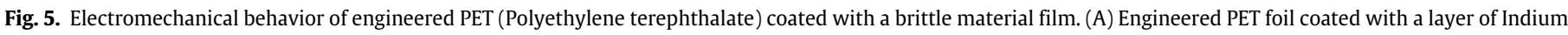

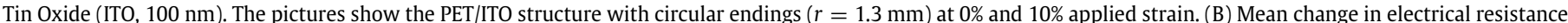

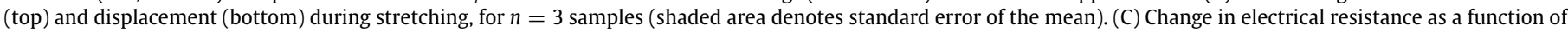

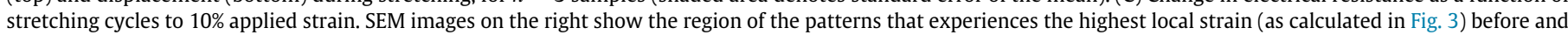
after 100,000 cycles.

We used a $75 \mu \mathrm{m}$ thick PET carrier substrate, patterned it with Y motifs with circular endings, and then coated the plastic with 100 $\mathrm{nm}$ thick ITO (Fig. 5(A)). The dogbone constructs were stretched to $10 \%$ applied strain as described in the previous section (Fig. 5(B)).

The electrical resistance of the ITO film remained finite until $70 \%$ applied strain and displayed an overall increase of $\sim 10$ times (from $0 \%$ to $70 \%$ strain) (Fig. 5(C)). Contrary to the ductile films, electrical damage and failure occurred before mechanical fracture in the ITO/PET constructs. For example, at $40 \%$ applied strain, limited force was measured in the sample but its electrical resistance had already increased by a factor of 5 .

The ITO/PET constructs were also cycled 100,000 times at $10 \%$ applied strain (Fig. 5(D)). Within the first few cycles, the resistance increased by a factor of 6 , possibly due to the formation of small cracks in the ITO film. However, after this initial jump, the resistance remained very stable and, had increased by less than a factor of 2 by the end of the 100,000 cycles.

It was also interesting to observe that following the first cycles, the resistance variation at $0 \%$ and $10 \%$ strain was much smaller in the ITO sample than that in the Pt film. SEM imaging revealed minimal (if any) cracking of the ITO film indicating the strain relief engineered with the Y motifs is very efficient.

\section{Conclusion}

In summary, we have demonstrated an efficient approach to engineer elasticity in films supported by a plastic carrier. The symmetry of the Y motifs allows for multi-axial stretchability, which few alternative techniques enable. Strain relief relies on out-ofplane bending of the substrate ligaments and re-organization of the Y motif openings. By optimizing the Y motifs endings with disks, the strain within the film's plastic ligament can be further minimized and offer larger elasticity. Other motifs could be explored as kirigami patterns. However, we showed here that the $Y$ motifs are sufficient to allow for conductors with combined multiaxial stretchability and low electrical resistance. Once coated with thin conducting film(s), the multilayered construct can stretch reversibly while maintaining reliable electrical conductivity. Patterning the substrate with replicated Y motifs is compatible with thin-film electronics processing. The concept can therefore be applicable to the broad range of electronic materials.

This study focused mainly on understanding the effect of the geometrical parameters of the $Y$ shaped cuts on the electromechanical characteristics of conductive films supported by a plastic foil. Even though for the purpose of this study the dimensions were in the mm-scale, we anticipate our kirigami-inspired approach can be scaled to the micro- and nano-scale. The engineered substrate may then contain patterned thin film devices, which could be encapsulated in stretchable silicones. We hypothesize that when reducing the size of our Y-shape cuts, the out-of-plane deflection will be small and not hindered by the encapsulation material, similarly to the microcracked gold films, which are stretchable even when fully encapsulated in PDMS [2,13]. This approach will have applications in a wide range of flexible and stretchable devices.

\section{Acknowledgments}

The authors would like to acknowledge the Bertarelli Foundation for funding this research and the Fulbright / Swiss Government scholarship to CMT. The authors are also very thankful to Prof. Z. Suo and Dr. Q. Liu for stimulating discussion and guidance in finite element modeling. Finally, the authors would like to thank Robin Amacher for giving us access and helping us characterizing samples with the axial-torsional servohydraulic testing machine.

\section{References}

[1] T. Sekitani, Y. Noguchi, K. Hata, T. Fukushima, T. Aida, T. Someya, A rubberlike stretchable active matrix using elastic conductors, Science (80-. ) 321 (5895) (2008) 1468-1472.

[2] I.R. Minev*, P. Musienko*, A. Hirsch, Q. Barraud, N. Wenger, E.M. Moraud, J. Gandar, M. Capogrosso, T. Milekovic, L. Asboth, R.F. Torres, N. Vachicouras, Q. Liu, N. Pavlova, S. Duis, A. Larmagnac, J. Vörös, S. Micera, Z. Suo, G. Courtine, S.P. Lacour, Electronic dura mater for long-term multimodal neural interfaces, Science (80-. ). 347 (6218) (2015) 159-163. 
[3] Z. Chen, W. Ren, L. Gao, B. Liu, S. Pei, H.-M. Cheng, Three-dimensional flexible and conductive interconnected graphene networks grown by chemical vapour deposition, Nature Mater. 10 (6) (2011) 424-428.

[4] A. Hirsch, H.O. Michaud, A.P. Gerratt, S. de Mulatier, S.P. Lacour, Intrinsically stretchable biphasic (solid-liquid) thin metal films, Adv. Mater. (2016) 1-6.

[5] D.S. Gray, J. Tien, C.S. Chen, High-conductivity elastomeric electronics, Adv. Mater. 16 (5) (2004) 393-397.

[6] Q. Ma, H. Cheng, K.I. Jang, H. Luan, K.C. Hwang, J.A. Rogers, Y. Huang, Y. Zhang, A nonlinear mechanics model of bio-inspired hierarchical lattice materials consisting of horseshoe microstructures, J. Mech. Phys. Solids 90 (2016) 179202.

[7] V. Kunin, S. Yang, Y. Cho, P. Deymier, D.J. Srolovitz, Static and dynamic elastic properties of fractal-cut materials, Extreme Mech. Lett. 6 (2016) 103-114.

[8] Y. Zhang, Z. Yan, K. Nan, D. Xiao, Y. Liu, H. Luan, H. Fu, X. Wang, Q. Yang, J. Wang, W. Ren, H. Si, F. Liu, L. Yang, H. Li, J. Wang, X. Guo, H. Luo, L. Wang, Y. Huang, J.A. Rogers, A mechanically driven form of Kirigami as a route to 3D mesostructures in micro/nanomembranes, Proc. Natl. Acad. Sci. 112 (38) (2015) 11757-11764.
[9] C.F. Guo, Q. Liu, G. Wang, Y. Wang, Z. Shi, Z. Suo, C. Chu, Z. Ren, Fatigue-free, superstretchable, transparent, and biocompatible metal electrodes, Proc. Natl. Acad. Sci. 112 (40) (2015) 12332-12337.

[10] T.C. Shyu, P.F. Damasceno, P.M. Dodd, A. Lamoureux, L. Xu, M. Shlian, M. Shtein, S.C. Glotzer, N.a. Kotov, A kirigami approach to engineering elasticity in nanocomposites through patterned defects, Nature Mater. 14 (8) (2015) 785789.

[11] O. Graudejus, P. Görrn, S. Wagner, Controlling the morphology of gold films on poly(dimethylsiloxane), ACS Appl. Mater. Interfaces 2 (7) (2010) 1927-1933.

[12] S.P. Lacour, S. Wagner, Z. Huang, Z. Suo, Stretchable gold conductors on elastomeric substrates, Appl. Phys. Lett. 82 (15) (2003) 2404.

[13] S.P. Lacour, D. Chan, S. Wagner, T. Li, Z. Suo, Mechanisms of reversible stretchability of thin metal films on elastomeric substrates, Appl. Phys. Lett. 88 (20) (2006) 204103.

[14] S.E. Schausberger, R. Kaltseis, M. Drack, U.D. Cakmak, Z. Major, S. Bauer, Costefficient open source desktop size radial stretching system with force sensor, IEEE Access 3 (2015) 556-561. 